\title{
Ultrasound Guided Transverse Carpal Ligament Fenestration for Carpal Tunnel Syndrome: A Case Series
}

\author{
George C. Chang Chien, DO' 1 , Yu M. Chiu, DO², Ramsin M. Benyamin, MD³, and Frank Falco, MD
}

Carpal tunnel syndrome is caused by compression of the median nerve under the transverse carpal ligament. We present a description of an ultrasound-guided (USG) percutaneous technique of the transcarpal tunnel and provide a discussion to the evidence in utilizing a minimally invasive technique as an alternative to open and endoscopic carpal tunnel release.

USG percutaneous transverse carpal ligament fenestration is a quick, and relatively simple office based treatment for carpal tunnel syndrome. The use of ultrasound provides satisfactory visualization for the procedure and requires very little time and effort for setup. Here, in this case series, we detail the procedure and the results from our follow-up.

This report details a case series of 2 patients with confirmed electrodiagnostic findings of mild to severe median neuropathy at the wrist in a university hospital outpatient pain management center. USG percutaneous fenestration of the transcarpal ligament was performed under local anesthesia. Patients were followed up from 4 to 20 weeks.

It was found in patient 1 , a greater than $60 \%$ reduction in pain at 20 weeks follow-up, and patient 2 had at least $50 \%$ reduction in pain at 8 weeks follow-up. Both patients had a minimum of $50 \%$ improvement in pain and sensory disturbance and without any adverse events.

The main advantage of USG transverse carpal ligament fenestration is that it is an office based procedure, requiring local anesthesia only. Minimally invasive USG transcarpal ligament fenestration, in select patients, may be an appropriate tool and even prove to be a surgery-sparing modality. The limitations of this study includes no case-control, small sample size, and the short term follow-up. In conclusion, this case series presents an alternative to a more invasive and costlier procedure including open and endoscopic carpal tunnel release, usually performed in a surgical suite. USG percutaneous transverse carpal ligament fenestration is a quick, and relatively simple office based treatment for carpal tunnel syndrome. The use of ultrasound provides satisfactory visualization for the procedure and requires very little time and effort for setup.

Key words: Ultrasound guided, transverse carpal ligament, fenestration, carpal tunnel syndrome
Carpal tunnel syndrome (CTS), also known as median mononeuropathy at the wrist, is caused by compression of the median nerve under the transverse carpal ligament (TCL) at the base of the palm (1). It

From : ${ }^{1}$ Ventura County Medical Center Pain Management, Ventura, CA, Southern California University of Health Sciences Center for Regenerative Medicine, Whittier, CA; ${ }^{2} \mathrm{NYU}$ Physical Medicine and Rehabilitation, New York, NY; ${ }^{3}$ Millennium Pain Center, Bloomington, IL, University of Illinois, Urbana-Champaign, IL; ${ }^{4}$ Mid Atlantic Spine and Pain Physicians, Newark, DE, Pain Medicine Fellowship Program, Temple University Hospital, Philadelphia, PA

Author for correspondence: Yu M. Chiu, DO

Address: NYU Physical Medicine and Rehabilitation, 240 E. 38th St, 15th Floor, Rm 15-82, New York, NY 10016-2708

E-mail:Yu.Chiu@nyumc.org is the most common entrapment neuropathy, with a prevalence ranging from $2.7-5.8 \%$ of the general population, with a mean annual crude incidence of 329 cases per 100,000 people (standardized incidence of 276). Patients with CTS report pain and paresthesia along the distribution of the median nerve (i.e., thumb, index finger, and middle finger), without any symptoms in the dorsum or palm of the hand. Patients also report loss of grip strength, nighttime awakening from pain, and history of shaking the hand or flicking the wrist to alleviate discomfort (Flick sign) (2).

In a 2011 study, workers with CTS were found to miss work for a median of 28 days, costing a wage loss of between $\$ 45,000$ and $\$ 89,000$, during and 6 years after treatment (3). 
Diagnosis of CTS should be based on a combination of history, physical examination, and results of electrophysiological studies. Various diagnostic tests include: Tinel's sign, Phalen's sign, square wrist sign, closed fist sign, flick sign (93\% sensitivity, 96\% specificity), Katz hand diagram, flexion and extension of wrist test, pressure provocation test, and tourniquet test. Nerve conduction studies (NCS) are useful in identifying and quantifying the magnitude of median mononueropathy, even though they are associated with false positive and false negative results. The combination of physical examination and electrodiagnostic evidence greatly increases diagnostic accuracy and specificity (4).

Current guidelines from the American Academy of Orthopedic Surgeons for CTS intervention include a course of nonsurgical treatment and early surgical intervention with clinical evidence of median nerve denervation or when patient elects. Guidelines further state nonsurgical treatment or surgery is suggested when current treatment fails to resolve symptoms within 2 to 7 weeks. Furthermore, local steroid injection or splinting is suggested before considering surgery, and the recommendation for surgical treatment is the complete division of the flexor retinaculum (5).

Pathophysiology of CTS is due to multifactorial causes, including nerve compression and traction disorders of the intraneural microcirculation, direct lesioning of the myelin sheath and axon, and alteration in the supporting connective tissue. Increased carpal tunnel pressure is thought to cause ischemic compression of the median nerve. The changes in microvascular structure of the nerve and the biochemical disturbances leads to reduction in the enodoneurial blood flow. Edema from increased permeability of the endoneurial vessels leads to increased diffusion distance for oxygen causing hypoxia, leading to upregulation of angiogenic factors and ultimately axonal degeneration of median nerve and neuritis (6).

Peripheral nerve regeneration is a relatively slow process, proceeding at a rate of $1 \mathrm{~mm} /$ day in humans. One study showed reinnervation of the thenar eminence by the median nerve after carpal tunnel release (CTR) of at least 12 months (7). In a cohort study of 45 patients with mild, moderate, and severe CTS, individuals were treated with CTR and a postoperative electrophysiologic evaluation was performed 2 weeks, 2 months, and 6 months after surgical de- compression. The authors found that the mild group had significant electrophysiologic improvement immediately after decompression. The moderate group showed marked improvement in nerve conduction values in all cases. In the severe group, electrophysiologic improvement was seen, but normalization of electrophysiologic test values were only possible in a few patients (8). Extrapolating from this study, it would be safe to assume 6 months to be adequate for repeat electrodiagnostic testing.

\section{Carpal Tunnel Anatomy}

The carpal tunnel serves as the conduit for the nerves, tendons, and vessels from the arm to the hand. The carpal bones that create the tunnel are oriented in 2 transverse rows. The proximal row, creates the carpal tunnel inlet, while the distal row creates the carpal tunnel outlet.

The median nerve enters the wrist, along with 9 finger flexor tendons (the flexor pollicis longus, the 4 flexor digitorum superficialis, and the 4 flexor digitorum profundus), through the carpal tunnel. The floor and sides of the tunnel are composed of carpal bones, while the flexor retinaculum, or TCL, forms a fibrous band of tissue that arches over the carpal bones of the hands, covering them and forming volar boundary of the carpal tunnel. On the ulnar side, the flexor retinaculum attaches to the pisiform bone and the hook of the hamate bone. On the radial side, it attaches to the scaphoid bone, and to the medial part of the trapezium bone. The ulnar artery and ulnar nerve, and the cutaneous branches of the median and ulnar nerves, pass on top of the flexor retinaculum. On the radial side of the retinaculum is the tendon of the flexor carpi radialis (Fig. 1).

The TCL acts as a pulley for the flexor tendons, an anchoring site for the thenar and hypothenar muscles, stabilizes and provides wrist proprioception (9). Utilizing silicone casting technique to examine TCL morphology in cadaveric hands, Pacek et al (10), found that the TCL has variable thickness ranging from 1.3 to $3 \mathrm{~mm}$, with a mean thickness $2.1 \mathrm{~mm}$. The midline and ulnar portions of the TCL are thickest distally, while the radial portion is the thickest proximally. The length of the TCL in the longitudinal direction varies between 12.5 to $17.7 \mathrm{~mm}$, shortest in the midline with an average of $14.4 \mathrm{~mm}$.

Vasculature in this area include both the deep and 
superficial palmar arch, consisting of the terminal parts of the radial and ulnar arteries. The deep palmar arch lies upon the bases of the metacarpal bones, just distal to the carpal tunnel, whereas the superficial palmar arch is more distal in the palm of the hand.

\section{TCL Fenestration}

The act of perforating a ligament or tendon is termed, fenestration. Although carpal tunnel injections are commonly performed by surface anatomical landmarks and palpation, the use of ultrasound guidance provides greatly increased accuracy and has been demonstrated to provide improved outcomes over landmark based carpal tunnel injections $(11,12)$. Ultrasound-guided (USG) fenestration occurs once the targeted area is identified via ultrasound and the needle is inserted along the long axis of the tissue. The needle is then passed into the target, withdrawn, redirected, and passed into the targeted tissue. The procedure is terminated when the targeted area is treated and feels soft during needle advancement. Contraindications to USG fenestration includes patient with bleeding disorder, anticoagulated, and presence of local infection (13).

We describe our experience with USG TCL fenestration in 2 patients with symptomatic median neuropathy at the wrist (Table 1).

\section{Description of Technique}

The patient is seated with their wrist in supination. A rolled up towel is placed under the dorsal wrist to

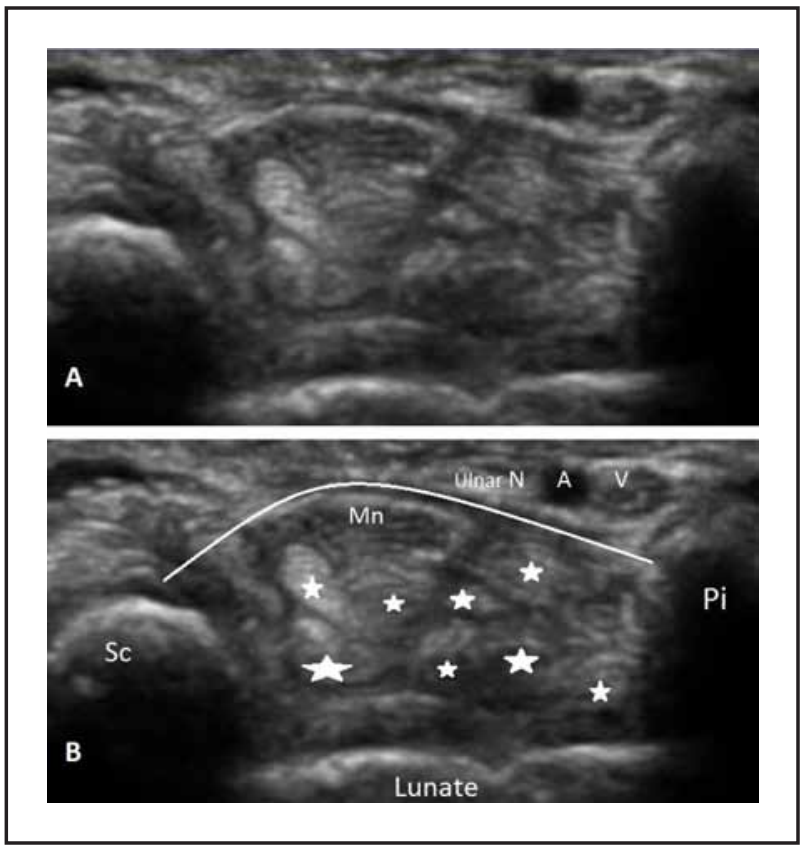

Figs. 1A and 1B. Ultrasound cross sectional anatomy of the carpal tunnel at the level of the pisiform bone.

$\mathrm{Sc}=$ scaphoid bone; $\mathrm{Pi}=$ Pisiform bone; Ulnar NAV = ulnar nerve, artery, vein; $\mathrm{Star}=$ flexor tendons. Flexor carpi radialis and Flexor pollicis longus not labeled

position the wrist in slight extension. The skin overlying the carpal tunnel is prepared and draped in the usual sterile fashion. Utilizing a high frequency linear transducer, covered in a sterile probe cover, and sterile gel, the carpal tunnel is visualized to identify the anatomy and appropriate entry point. Utilizing the pisiform bone as the major landmark associated

Table 1. Summary of patients.

\begin{tabular}{|c|c|c|c|c|c|}
\hline Patient & $\begin{array}{l}\text { Electrodiagnostic } \\
\text { Findings }\end{array}$ & Conservative Care & $\begin{array}{c}\text { Results Post Transcarpal Ligament } \\
\text { Fenestration }\end{array}$ & Follow-up & $\begin{array}{c}\text { Adverse } \\
\text { effects }\end{array}$ \\
\hline 1 & $\begin{array}{c}\text { Median sensory NCS } \\
\text { (transcarpal): prolonged } \\
\text { peak, SNAP value less than } \\
50 \% \text { reference value. } \\
\text { Median motor NCS: } \\
\text { prolonged distal latency, } \\
\text { normal CMAP amplitude. } \\
\text { NCV }=50 \mathrm{~m} / \mathrm{sec}\end{array}$ & $\begin{array}{l}\text { Wrist splint, } \\
\text { occupational therapy, } \\
\text { NSAIDs, } \\
\text { lifestyle modifications, } \\
\text { corticosteroid } \\
\text { injection. }\end{array}$ & $\begin{array}{l}\text { Greater than } 60 \% \text { improvement in pain, } \\
\text { sensory disturbance at all follow-up } \\
\text { periods. } \\
\text { Decision not to proceed with carpal tunnel } \\
\text { release. }\end{array}$ & $\begin{array}{l}8,12, \text { and } 20 \\
\text { weeks }\end{array}$ & None \\
\hline 2 & $\begin{array}{l}\text { Median sensory NCS } \\
\text { (transcarpal) absent. } \\
\text { Median motor NCS: } \\
\text { prolonged distal latency, } \\
\text { Reduced CMAP amplitude. } \\
\text { NCV }=39 \mathrm{~m} / \mathrm{sec}\end{array}$ & $\begin{array}{l}\text { Wrist splint, } \\
\text { occupational therapy, } \\
\text { NSAIDs, } \\
\text { hydrocodone, } \\
\text { corticosteroid } \\
\text { injection. }\end{array}$ & $\begin{array}{l}\text { Greater than } 50 \% \text { improvement in pain and } \\
\text { sensory disturbance. } \\
\text { Improvement in pain at } 4,8 \text { week follow- } \\
\text { up. Return to "near" baseline pain at } 12 \\
\text { week follow-up. } \\
\text { Decision to proceed with surgical carpal } \\
\text { tunnel release. }\end{array}$ & $4,8,12$ weeks & None \\
\hline
\end{tabular}

$\mathrm{SNAP}=$ Sensory nerve action potential; $\mathrm{CMAP}=$ Compound motor action potential; $\mathrm{NCV}=$ Nerve conduction velocity; NSAID $=$ nonsteroidal anti-inflammatory drug 
with the carpal tunnel inlet, the contents of the carpal tunnel are identified. A 25-gauge, 1.5 inch needle enters the skin over the entry point between the ulnar neurovascular bundle and the median nerve. One $\mathrm{mL}$ of $1 \%$ preservative-free lidocaine is injected into the superficial skin under direct ultrasound visualization. The needle is then advanced under visualization to pierce the TCL, while avoiding sensitive structures. One to $2 \mathrm{~mL}$ of lidocaine is injected to hydrodissect the median nerve from the flexor tendons and TCL resulting in circumferential injectate cushioning the median nerve (Fig. 2). A 22-gauge, 1.5 inch needle is then introduced, in-plane with the ultrasound probe, through the anesthetized tissues and utilized to gently fenestrate the overlying TCL, starting from the ulnar portion and purposefully fenestrating towards to radial aspect. The ultrasound probe is then translated to the distal row of carpal bones where the fenestration procedure is repeated. The procedure is terminated when most areas of the ligament have been fenestrated. The ligament at this point will demonstrate reduced resistance to needle advancement. It is important to monitor for the superficial, and deep palmar arches and branches of the median or ulnar nerves. Care is also made not to exit the skin during fenestration to avoid creating multiple, unnecessary puncture wounds. After satisfactory fenestration of the TCL, a mixture of corticosteroid and local anesthetic solution can provide pain relief from the procedure and to treat the median neuropathy. In our 2 cases, the solution consisted of a mixture of $40 \mathrm{mg}$ of triamcinolone, and $1 \mathrm{~mL}$ of $1 \%$ lidocaine.

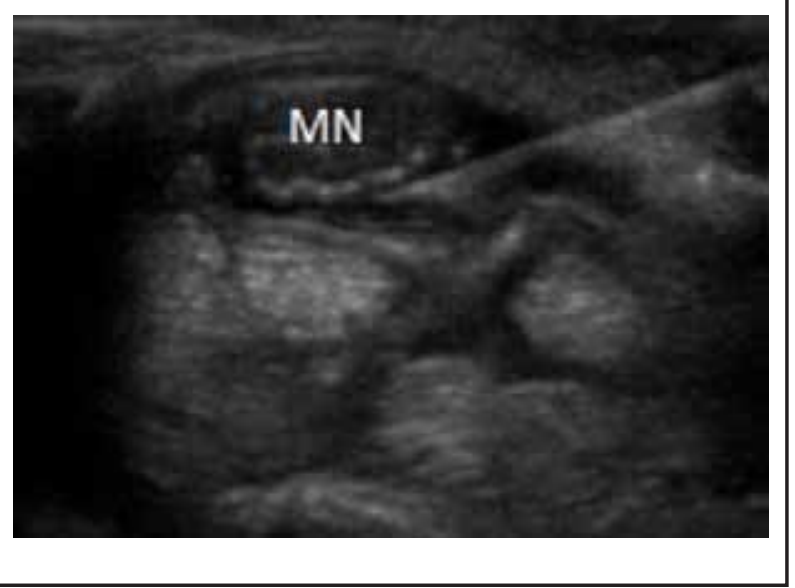

Fig. 2. Circumferential injectate surrounding the median nerve.

\section{Patient 1:}

A 50 year-old woman presented with left-sided median neuropathy at the wrist. She had previously undergone CTR in her right-hand, which halted progression of worsening disease although she had persistent weakness, and reduced sensation to light touch. Electrodiagnostic evaluation demonstrated mild to moderate median neuropathy at the wrist. Previous conservative therapy including wrist splinting, occupational therapy, and medication management failed to resolve her symptoms. She underwent USG injection of her left carpal tunnel with $40 \mathrm{mg}$ of triamcinolone and $1 \mathrm{~mL}$ of $1 \%$ lidocaine, which provided $80 \%$ pain relief lasting for 6 weeks. CTR was recommended by the surgical team. Based on patient preference, the decision was made to undergo percutaneous fenestration of her TCL prior to surgical option (Fig. 3).

\section{Patient 2:}

A 64 year-old woman presented with median neuropathy at the right wrist. She had weakness, thenar eminence flattening, reduced sensation to light touch, and reduced grip strength. Electrodiagnostic evaluation demonstrated moderate to severe median neuropathy at the wrist. Previous conservative therapy including wrist splinting, occupational therapy, and medication management, failed to resolve her symptoms. She underwent USG injection of her left carpal tunnel with $40 \mathrm{mg}$ of triamcinolone and $1 \mathrm{~mL}$ of $1 \%$ lidocaine, which provided $70 \%$ pain relief lasting for 4 weeks. Pending surgical evaluation, the patient preferred to undergo percutaneous fenestration of her TCL (Fig. 4).

\section{RESULTS}

The 2 presented cases provide an interesting demonstration of minimally invasive USG interventions in the treatment of persistent, symptomatic median neuropathy at the wrist. Patient 1 responded very well to percutaneous TCL fenestration, with greater than $60 \%$ reduction in pain that persisted up to the 20 week follow-up. Although she had been recommended for open carpal tunnel release (OCTR), she opted not to proceed with surgical release.

Patient 2 had a $50 \%$ improvement in pain, sensory disturbances, and pain that lasted greater than 8 weeks, but to "near" baseline pain at 12 week follow- 

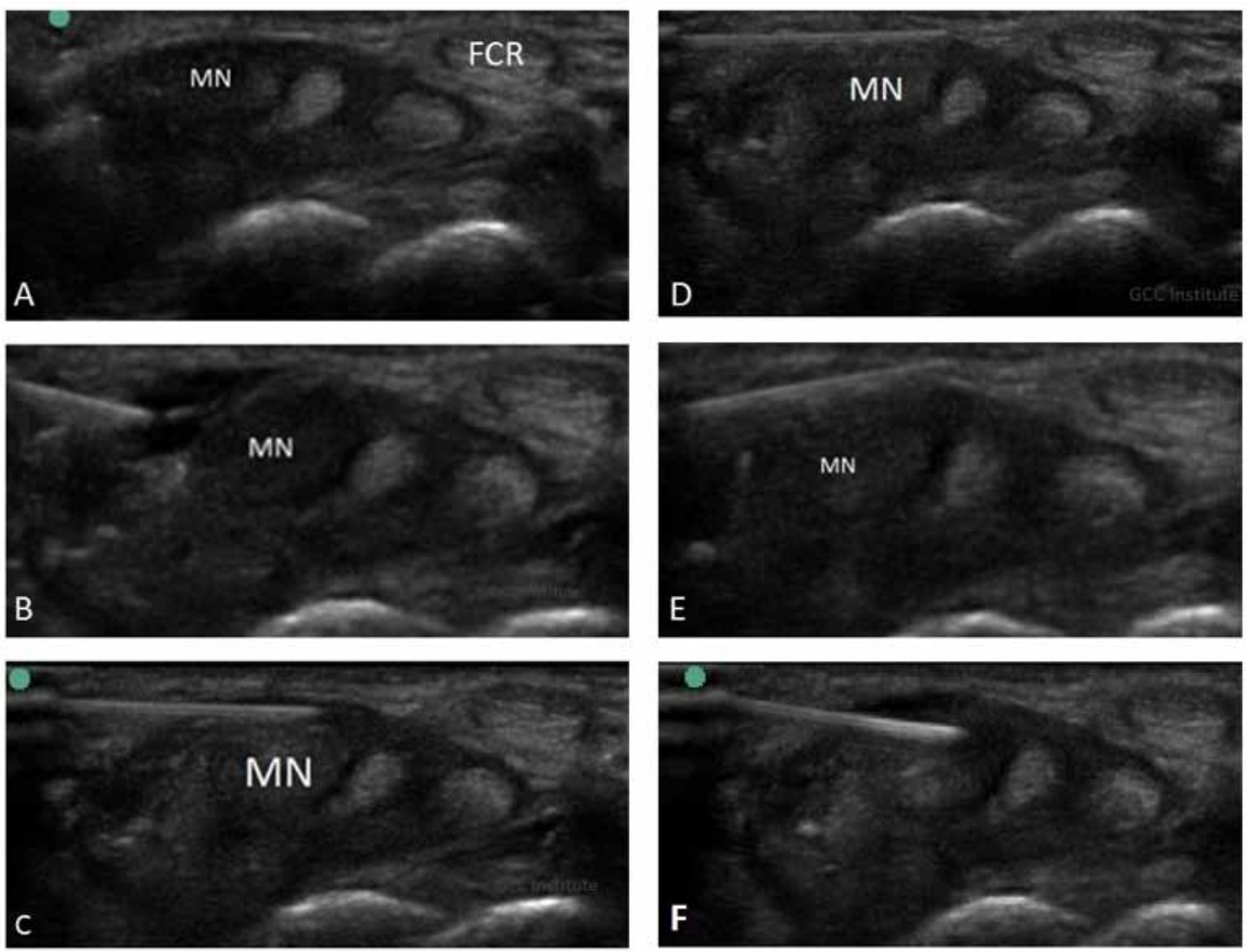

Fig. 3. USG percutaneous fenestration of the transcarpal ligament, case 2. A) Scan of the carpal tunnel prior to procedure. B and C) Hydrodissection of the median nerve away from the flexor tendons, and away from the transcarpal ligament. D and E) Repeated fenestration of the transcarpal ligament. F) Injectate separating the median nerve from the transcarpal ligament. Utilizing the edge of the needle to depress the median nerve.

$\mathrm{MN}=$ Median Nerve; FCR $=$ flexor carpi radialis

up. She subsequently underwent surgical CTR to treat her median neuropathy.

No side effects or adverse outcomes were identified in either case.

\section{DISCUSSION}

Conservative management of CTS includes lifestyle modification, oral medications, splinting, corticosteroid injections. CTR is a commonly performed operative technique for CTS when conservative treatment has failed. CTR can be performed both as OCTR, or endoscopic carpal tunnel release (ECTR). Thurston et al (14), reported that OCTR is a safer and more preferable option when compared to ECTR. Boeckstyns et al (15), published an analysis of 9,516 ECTR and 1,203 OCTR and found both methods were compa- rable in rate of irreversible nerve damage, $0.3 \%$ and $0.2 \%$, respectively. Limitations in OCTR, including scar tenderness, pillar pain, weakness, and delay in return to work, resulted in the development of ECTR in the late 1980s. The average return to work time is 54 days in OCTR and 28 days in ECTR. ECTR resulted in rapid return of strength and function, however concerns for risks of median nerve injury and incomplete release, as well as a narrow view of the surgical field via endoscopic probe, a steep learning curve, high device cost, and significant setup time and effort, prompted the use of USG CTR (16).

Both OCTR and ECTR are typically performed with either general or regional anesthesia. During a 5-year time period, utilizing the Pearl Diver Patient Records Database, from $2007-2011$ reported that $80.5 \%$ of 

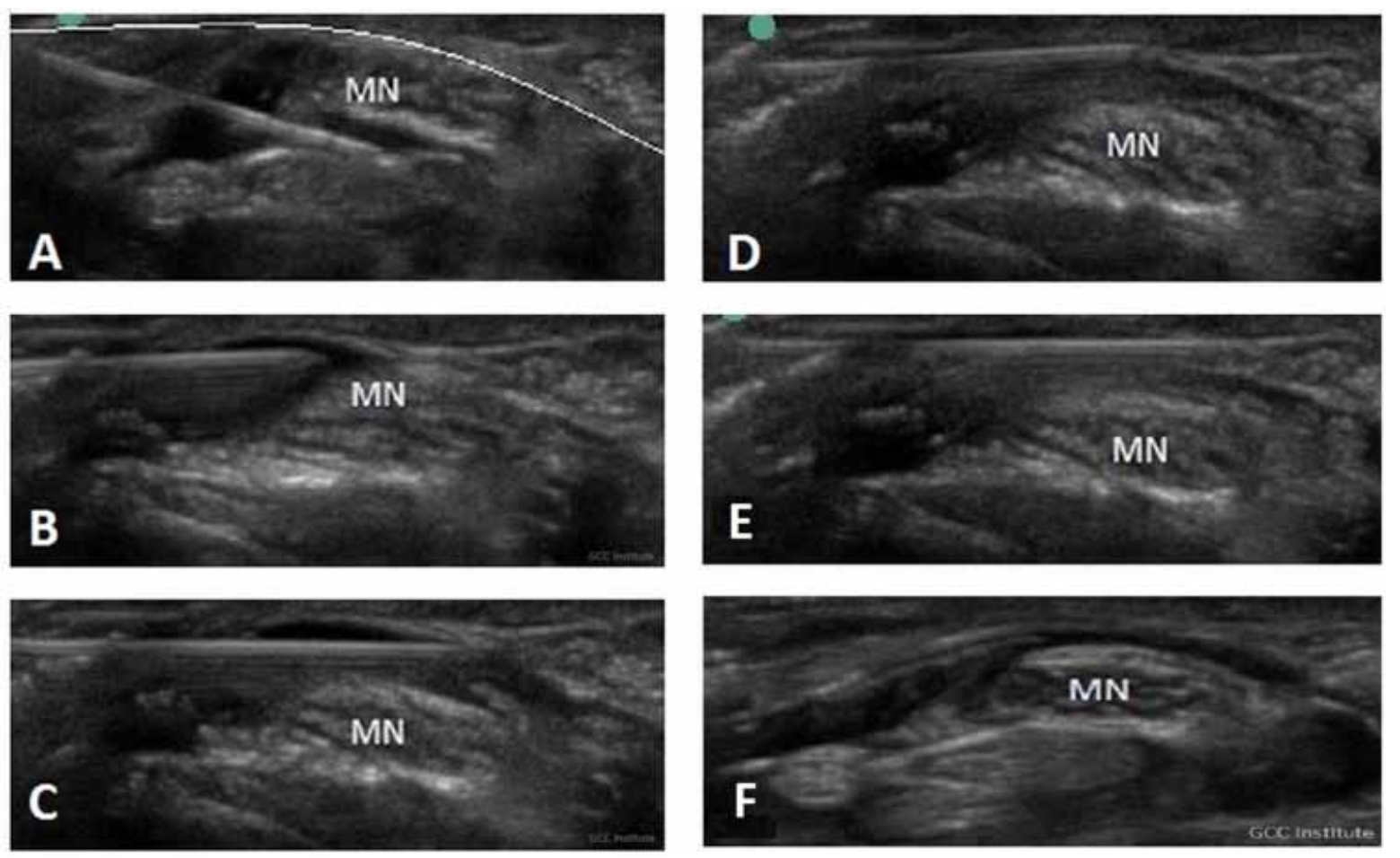

Fig. 4. USG percutaneous fenestration of the transcarpal ligament. A and B) Hydrodissection of the median nerve away from the flexor tendons, deep to the MN, and transcarpal ligament (outlined with white line) superficial to the MN. C-E) Repeated fenestration of the transcarpal ligament. F) Final image of the enlarged median nerve, with circumferential injectate.

$\mathrm{MN}=$ Median Nerve. Transcarpal ligament (outlined with white line)

CTR were performed under general or regional anesthesia compared to $19.5 \%$ using local anesthesia. $83.9 \%$ of all CTR were performed in an open fashion, compared to $16.1 \%$ performed using an endoscopic technique. Cost analysis showed endoscopic surgery was on average $\$ 794$ more expensive than open surgery, and general or regional anesthesia was $\$ 654$ more costly than local anesthesia (17).

More recent percutaneous methods for CTR have been developed, but did not demonstrate any advantages when compared to the open procedure in respect to improvement in pain, numbness, or patient satisfaction (18). In a study published in 2015, authors utilized a percutaneously looped thread transection of the TCL. A limitation to this particular approach was the mini tools required for repetitive cutting motions to divide the TCL, the increased risk of technical errors, iatrogenic injuries, and incomplete release (16).
McShane et al (1), reported a study of 13 patients undergoing USG percutaneous needle release of the TCR with a 18-gauge needle. These authors reported a significantly smaller cross-sectional area of the median nerve compared to pretreatment values, significantly fewer symptoms, less functional impairment, and an improved hand diagram score, with $86 \%$ reported satisfaction with their procedure. Furthermore, no procedure related infections or nerve injuries were reported.

Recently a case report showed the use of USG fenestration of the carpal ligament using a double needle approach that occurred after the inability to hydrodissect the median nerve away from the retinaculum. The double needle approach allowed for a successful hydrodissection of the overlying carpal ligament to allow for safe fenestration with the first needle, without risk to the underlying nerve (19). 
In a 2015 cadaver study, 401 performed tenotomies were assessed, of which $72 \%$ were complete, $24.9 \%$ partial, and $2.7 \%$ failed. Of the failed, adjacent lesions occurred $2 \%$ of the time, lesions in tendons or muscles in $1 \%$ of the time, lesions in nerves $0.7 \%$ of the time, and lesions in a vessel $0.2 \%$ of the time. The authors suggested that percutaneous needle technique is effective in rupturing the desired tendon with few injuries to adjacent structures, and the results suggest it is safe to carry out on patients (20). Figure 5 details the anatomy of the pertinent structures of the hand in both anteroposterior and tunnel view.

The main advantage of USG TCL fenestration is that it is an office based procedure, performed under local anesthesia. This demonstrates a significant reduction in utilization of resources as regional or general anesthesia is not necessary. Nonetheless, there are no studies to date that compare this percutaneous approach to the traditional CTR.

Our 2 patients demonstrated successful fenestration of the ligament utilizing a 22-gauge needle. patient 1 obtained significant benefit in pain reduction, and opted not to proceed with surgical intervention. We surmise that one reason for the outcome disparity in these 2 patients was due to the difference in baseline severity of their CTS, whereas patient 1 had moderate CTS symptoms and patient 2 had severe CTS.

\section{CONCLUSION}

USG percutaneous TCL fenestration is a quick, and relatively simple office based treatment for CTS. Ultrasound provides satisfactory visualization for the procedure and requires very little time and effort for setup.

This approach is not without limitations, and likely will only be useful in select number of patients with relatively mild disease. Fenestration with a needle does not create transection across the TCL. Importantly, we did not measure pre- and post-procedure median nerve cross-sectional areas, measure functional outcomes, or repeat electrodiagnostic testing after the intervention. We postulate that the improved outcomes in patient 1 were due to her mild to moderate symptoms.

Minimally invasive USG TCL fenestration may be an appropriate tool for select patients and may even prove to be a surgery-sparing modality in select patients. Further well-conducted prospective studies comparing these treatment modalities is warranted to identify the best protocols, and identify which patients may respond to this procedure. Further refinement of USG percutaneous TCL fenestration techniques may lead to a convenient, office based alternative to traditional CTR.
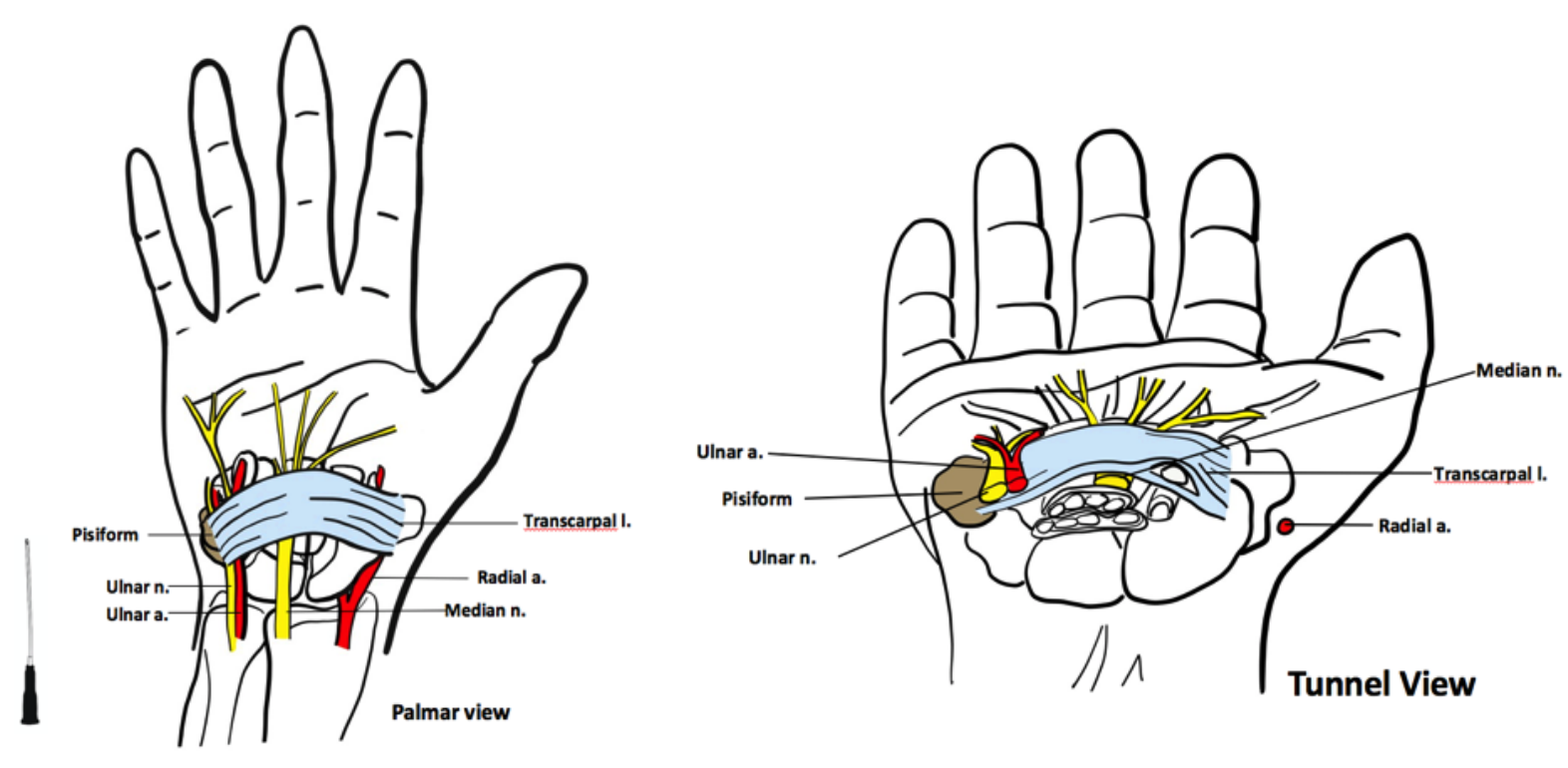

Fig. 5. Anatomy of hand anteroposterior and tunnel view.

Fig. created by Colette Lam, Charlotte, NC. CLamOriginals@gmail.com 


\section{REFERENCES}

1. McShane JM, Slaff S, Gold JE, Nazariam LN. Sonographically guided percutaneous needle release of the carpal tunnel for treatment of carpal tunnel syndrome. Preliminary report. J Ultrasound Med 2012; 31:1341-1349.

2. LeBlanc KE, Cestia W. Carpal tunnel syndrome. Am Fam Physician 2011; 83:952-958

3. Foley M, Silverstein B, Pollissar N. The economic burden of carpal tunnel syndrome: long-term earnings of CTS claimants in Washington State. Am J Ind Med 2007; 50:155-172.

4. Aroori S, Spence RA. Carpal tunnel syndrome. Ulster Med J 2008; 77:6-17.

5. Keith MW, Masear V, Chung KC, Amadio PC, Andary M, Barth RW, Maupin K, Graham B, Watters WC 3rd, Turkelson CM, Haralson RH, Wies JL, McGowan R; American Academy of Orthopaedic Surgeons. American Academy of Orthopaedic Surgeons clinical practice guideline on the treatment of carpal tunnell syndrome. J Bone Joint Surg Am 2010; 92:218-219.

6. Aboonq MS. Pathophysiology of carpal tunnel syndrome. Neurosciences (Riyadh) 2015; 20:4-9.

7. Gordon T, Brushart TM, Chan KM. Augmenting nerve regeneration with electrical stimulation. Neurol Res 2008; 30:1012-1022.

8. Aulisa L, Tamburrelli F, Padua R, Romanini E, Lo Monaco M, Padua L. Carpal tunnel syndrome: Indication for surgical treatment based on electrophysiologic study. J Hand Surg Am 1998; 23:687-691.

9. Li ZM, Marquardt TL, Evans PJ, Seitz WH Jr. Biomechanical role of the transverse carpal ligament in carpal tunnel compliance. J Wrist Surg 2014; 3:227-232.

10. Pacek CA, Chakan M, Goitz RJ, Kaufmann RA, Li Zm. Morphological analysis of the transverse carpal ligament. Hand (NY) 2010; 5:135-140.

11. Eslamian F, Eftekharsadat B, Babaei-Ghazani A, Jahanjoo F, Zeinali M. A randomized prospective comparison of ultrasoundguided and landmark-guided steroid injections for carpal tunnel syndrome. J Clin Neurophysiol 2017; 34:107-113.

12. Ustun N, Tok F, Yagz AE, Kizil N, Korkmaz I, Karazincir S, Okuyucu E, Turhanoglu AD. Ultrasound-guided vs. blind steroid injections in carpal tunnel syndrome: A single-blind randomized prospective study. Am J Phys Med Rehabil 2013; 92:999-1004.

13. Chiavaras MM, Jacobson JA. Ultrasound-guided tendon fenestration. Semin Musculoskelet Radiol 2013; 17:85-90.

14. Thurston A, Lam N. Results of open carpal tunnel release: $A$ comprehensive, retrospective study of 188 hands. Aust N Z J Surg 1997; 67:283-288.

15. Boeckstyns ME, Sorensen AI. Does endoscopic carpal tunnel release have a higher rate of complications than open carpal tunnel release? An analysis of published series. J Hand Surg $\mathrm{Br}$ 1999; 24:9-15.

16. Guo D, Tang Y, Ji Y, Sun T, Guo J, Guo D. A non-scalpel technique for minimally invasive surgery: Percutaneously looped thread transection of the transverse carpal ligament. Hand (NY) 2015; 10:40-48.

17. Foster BD, Sivasundaram L, Heckmann N, Cohen JR, Pannell WC, Wang JC, Ghiassi A. Surgical approach and anesthetic modality for carpal tunnel release: A nationwide database study with health care cost implications. Hand (NY) 2017; 12:162-167.

18. Yeo KQ, Yeo EM. Comparison of the results of open carpal tunnel release and KnifeLight carpal tunnel release. Singapore Med J 2007; 48:1131-1135.

19. Hwang GE, Kumbar S, Stitik T, Georgy JS, Hanna H. Ultrasound-guided fenestration of the carpal ligament using a double-needle approach. Am J Phys Med Rehabil 2017; 96:e185e188.

20. Chesnel C, Genet F, Almangour W, Denormandie P, Parratte B, Schnitzler A. Effectiveness and complications of percutaneous needle tenotomy with a large needle for muscle contractures: A cadaver study. PLoS One 2015; 10:e0143495. 